LAWRENCE LIVERMORE NATIONAL LABORATORY

\title{
Biological Applications of Cryogenic Detectors
}

S. Friedrich

December 3, 2003

$10^{\text {th }}$ International Workshop on Low Temperature Detectors, Genoa, Italy, July 7-11, 2003 
This document was prepared as an account of work sponsored by an agency of the United States Government. Neither the United States Government nor the University of California nor any of their employees, makes any warranty, express or implied, or assumes any legal liability or responsibility for the accuracy, completeness, or usefulness of any information, apparatus, product, or process disclosed, or represents that its use would not infringe privately owned rights. Reference herein to any specific commercial product, process, or service by trade name, trademark, manufacturer, or otherwise, does not necessarily constitute or imply its endorsement, recommendation, or favoring by the United States Government or the University of California. The views and opinions of authors expressed herein do not necessarily state or reflect those of the United States Government or the University of California, and shall not be used for advertising or product endorsement purposes. 


\title{
Biological applications of cryogenic detectors
}

\author{
Stephan Friedrich* \\ Lawrence Livermore National Laboratory \\ 7000 East Ave., L-270, Livermore, CA 94550, U.S.A.
}

\begin{abstract}
High energy resolution and broadband efficiency are enabling the use of cryogenic detectors in biological research. Two areas where they have found initial application are X-ray absorption spectroscopy (XAS) and time-of-flight mass spectrometry (TOF-MS). In synchrotron-based fluorescence-detected XAS cryogenic detectors are used to examine the role of metals in biological systems by measuring their oxidation states and ligand symmetries. In time-of-flight mass spectrometry cryogenic detectors increase the sensitivity for biomolecule detection and identification for masses above $\sim 50 \mathrm{kDa}$, and thus enable TOF-MS on large protein complexes or even entire viruses. More recently, cryogenic detectors have been proposed as optical sensors for fluorescence signals from biomarkers. We discuss the potential for cryogenic detectors in biological research, as well as the challenges the technology faces.
\end{abstract}

Keywords: Cryogenic detectors; X-ray absorption spectroscopy; mass spectrometry; fluorescence resonace energy transfer

PACS: 85.25, 87.64, 07.75, 87.64.N

* Corresponding author. Tel.: +1-925-423-1527; fax: +1-925-424-5512; e-mail: friedrich1@1lnl.gov. 


\section{Introduction}

Cryogenic photon and particle detectors operating at temperatures below $1 \mathrm{~K}$ provide more than an order of magnitude higher energy resolution than conventional semiconductor detectors (though usually not as high as grating spectrometers), and an order of magnitude higher detection efficiency than grating spectrometers (though usually not as high as semiconductor detectors) [1]. Their development was initially driven by particle and astrophysics needs for dark matter searches and X-ray astronomy. More recently, cryogenic detectors have been applied in the biological sciences. Here we discuss an representative example of the role for cryogenic detectors in synchrotron-based X-ray spectroscopy on metalloenzymes, their application in time-of-flight mass spectrometry for large biomolecule identification, and their potential for single-molecule spectroscopy by fluorescence resonance energy transfer.

\section{X-ray Absorption Spectroscopy}

Synchrotron-based x-ray absorption spectroscopy (XAS) is widely used to characterize chemical states of elements in different coordination chemistries. Atomic energy levels are sampled with natural-linewidth-limited resolution by scanning the energy of a monochromatic X-ray beam through an absorption edge of the element of interest, and fine structure in the absorption spectra are indicative of the elemental oxidation and spin state, its bond symmetry and its ligand field strength. For dilute biological samples, the sensitivity is highest when recording the intensity of the corresponding x-ray fluorescence as a measure of absorption (fluorescence-detected XAS) [2]. This greatly reduces the number of background counts, provided that the fluorescence line of interest can be separated from neighbouring lines in multi-element specimens.

Cryogenic x-ray spectrometers [3] offer an advantage whenever conventional semiconducting detectors cannot resolve the fluorescence line of interest, and grating spectrometers lack the efficiency to collect data within an acceptable period of time. A short measurement time is crucial for biological samples that suffer from radiation damage.

Fluorescence-detected XAS in on active metal sites in proteins can be applied to examine their reaction mechanisms. One example is photosynthetic oxygen production in photosystem II (PSII) which occurs at a cluster of four Mn atoms. The absorption of four photons advances the Mn cluster through four distinct states, at which point oxygen is released and the cycle starts anew. Measuring the Mn oxidation state in the four states of the cycle constrains mechanistic models of the reaction mechanism. Cryogenic detectors are required to separate the weak Mn L fluorescence from the much stronger nearby oxygen line (figure 1a). The fluorescence-detected absorption spectrum (figure 1b) shows two main absorption features, the $\mathrm{L}_{3}$ edge at $\sim 642 \mathrm{eV}$ corresponding to the $2 \mathrm{p}_{3 / 2} \rightarrow 3 \mathrm{~d}$ transition and the $\mathrm{L}_{2}$ edge at $\sim 652 \mathrm{eV}$ corresponding to the $2 \mathrm{p}_{1 / 2} \rightarrow 3 \mathrm{~d}$ transition. The fine structure is characteristic for $\mathrm{Mn}$ in the oxidation state +2 , indicating that $\mathrm{Mn}$ in this dried and biologically inactive PSII sample is reduced to its most stable oxidation state +2 . Simulations of the absorption spectrum for 
$\mathrm{Mn}^{+3}$ and $\mathrm{Mn}^{+4}$ shown for comparison. The above experiment demonstrates both the strengths and the challenges using cryogenic detectors for X-ray spectroscopy on proteins. They enable spectroscopy on certain metal sites, but for very dilute or very sensitive samples like PSII higher detection efficiency is required. Using polycapillary X-ray focusing optics for increased solid angle coverage is not always possible, because it requires a small focal spot size $<100 \mu \mathrm{m}$ on the sample and consequently increases radiation damage. Increasing the array size and bringing it even closer to the sample than the $\sim 15 \mathrm{~mm}$ currently achieved will expand the range of possible experiments.

\section{Time-of-Flight Mass Spectrometry}

Time-of-flight mass spectrometry is a widely used technique to determine molecular mass distributions in composite samples. In biological and biomedical research it can identify proteins, protein complexes and their constituent subgroups. A short-pulse laser vaporizes and ionizes the sample at a start time $\mathrm{t}_{0}$. The ions, which have a mass $\mathrm{m}$ and total charge $\mathrm{q}$, are electrostatically accelerated by a field $\mathrm{E}$ and traverse a flight tube of length $\mathrm{L}$, at the end of which a fast microchannel plate detector records their time of arrival t. Heavier molecules travel more slowly, and the time of flight

$\mathrm{t}-\mathrm{t}_{0}=\mathrm{L}(\mathrm{m} / 2 \mathrm{qE})^{1 / 2}$

thus provides a measure of the particle mass.

Matrix-assisted laser desorption/ ionization and electrospray ionization are two comparably gentle techniques to produce ions that have enabled the launch of very large molecules without breaking them apart. However, microchannel plate detectors rely on secondary electron emission that becomes inefficient with increasing ion mass and typically limits their use in time-of-flight mass spectrometry to a range of $\sim 50 \mathrm{kDa}$.

Cryogenic detectors rely on the mass-independent detection of low-energy excitations like phonons or quasiparticles, and therefore have unity efficiency for arbitrarily high masses. Cryogenic detectors also provide information on the total energy deposited and can remove spectral ambiguities from ions with different charges $\mathrm{q}[4,5]$. This is especially useful in conjunction with electrospray ionization where a multitude of charge states are produced. Cryogenic detectors thus enable the identification of large proteins to examine their complex formation and ligand attachment. The analysis of DNA/ RNA and their fragments allows fast genotyping and the recognition of mutants. Even the detection of entire viruses for medical diagnostics or as an early-warning system against biological terrorism are possible. The potential of cryogenic detectors for mass spectrometry is currently leading to commercialization efforts [6].

There are, however, competing approaches to address the limitations of current technology. Secondary ion detectors and post-acceleration over a short distance at the end of the flight tube have been used to detect ions as heavy as 2 MDa. Multiply charged 
electrospray clouds can be partially deionized through interaction with a dilute gas, and some spectral ambiguities from different charge states can be addressed by post-processing in software. Also, the small size of cryogenic detectors reduces their advantage of high detection efficiency, given that refocusing the ion beam tends to degrade the mass resolution.

The impact cryogenic detectors will have on mass spectrometry will depend on gains in sensitivity for cryodetectors over that offered by improved conventional technology, and by the user-friendliness of their operation for non-expert users.

\section{Fluorescence Resonance Energy Transfer}

Fluorescence resonance energy transfer (FRET) is a technique to measure intermolecular distances and conformational changes in biological molecules [7, 8]. It uses two different fluorescent markers, one donor and one acceptor fluorophore, chosen such that the emission band of the donor overlaps with the absorption band of the acceptor (figure 2). An excited donor fluorophore can then resonantly transfer energy to the acceptor, provided that the two markers are in sufficiently close vicinity. The resonant transfer causes a decrease in the donor fluorescence intensity and the donor lifetime, as well as an increase in the acceptor fluorescence. The transfer efficiency E is given by

$$
\mathrm{E}=1 /\left(1+\left(\mathrm{R} / \mathrm{R}_{0}\right)^{6}\right)
$$

where $\mathrm{R}$ is the distance between the two fluorescence makers and $\mathrm{R}_{0}$ a distance characteristic for each donor-acceptor pair. Typically, $\mathrm{R}_{0}$ is in the range of 20 to $100 \AA$, a distance comparable to the diameter of most biological molecules. The steep dependence of the transfer efficiency on the interatomic distance $\mathrm{R}$ then makes FRET uniquely suited as a molecular ruler to measure distance changes and infer interactions between different proteins or conformational changes of single molecules.

The sensitivity of FRET experiments depends on the ratio of the fluorescence signal relative to the background noise, which is typically set by scattered light and background fluorescence. Highest sensitivity single molecule detection can be accomplished using confocal microscopes that excite an extremely small spot in the focal plane on the sample, and reduce the out-of-plane fluorescence background through pinhole apertures. The molecule is excited at the optical wavelength of maximum donor absorption and transfers its energy resonantly to the acceptor molecule depending on their relative distance. An interference filter selects the fluorescence wavelength to be monitored, and a fast avalanche photodiode records the intensity of the FRET fluorescence signal at single photon level. 2D images are obtained by scanning the focal spot across the sample, and subsequent tomographic volume rendering can be done. Avalanche photodiodes with single photon sensitivity are only available as single pixel detectors, which perfectly matches the confocal optical geometry.

Cryogenic detectors can enable single molecule FRET studies on systems whose fluorescence wavelength changes with the environmental conditions. Several markers, for 
instance, change their fluorescence wavelength as a function of local $\mathrm{pH}$ or ion concentration and can be used to monitor environmentally driven conformational changes. Interference filters have a limited bandpass, and splitting the fluorescence signal with dichroic filters and using separate interference filters for each fluorescence wavelength reduces the detection efficiency such that not enough photons can be collected within the finite lifetime of the fluorescence marker. An efficient broadband optical detector with a resolving power of at least 10 is needed. Also, often structural changes and environmental conditions are not static but fluctuate over millisecond time scales. Cryogenic detectors will enable dynamic studies of such processes, since their microsecond decay times allow spectral information can be extracted with tens of millisecond time resolution. The challenge that cryogenic detectors face is if the increase in sensitivity they provide will be considered sufficiently important by the biophysics community to justify the significant time and money investment in lowtemperature equipment.

\section{Acknowledgments}

We thank Daniel Prober and Matthias Frank for stimulating discussions. This work was performed under the auspices of the U.S. Department of Energy by University of California Lawrence Livermore National Laboratory under contract No. W-7405-Eng-48. 


\section{$\underline{\text { References }}$}

[1] For an overview of the field of cryogenic detectors, see these proceedings, and AIP Conf. Proc. 605 (2002).

[2] J. Jaclevic et al., Solid State Commun. 23 (1977) 679.

[3] S. Friedrich et al., Rev. Sci. Inst. 73 (2002) 1692.

[4] M. Frank et al., Mass. Spec Rev. 18 (1999) 155.

[5] G. Hilton et al., Nature 391 (1998) 672

[6] D. Twerenbold, US patent 5640010, http://www.comet.ch.

[7] P.R. Selvin, Nat. Struct. Biol 7 (2000) 730.

[8] S. Weiss, Science 283 (1999) 1676. 


\section{Figures:}
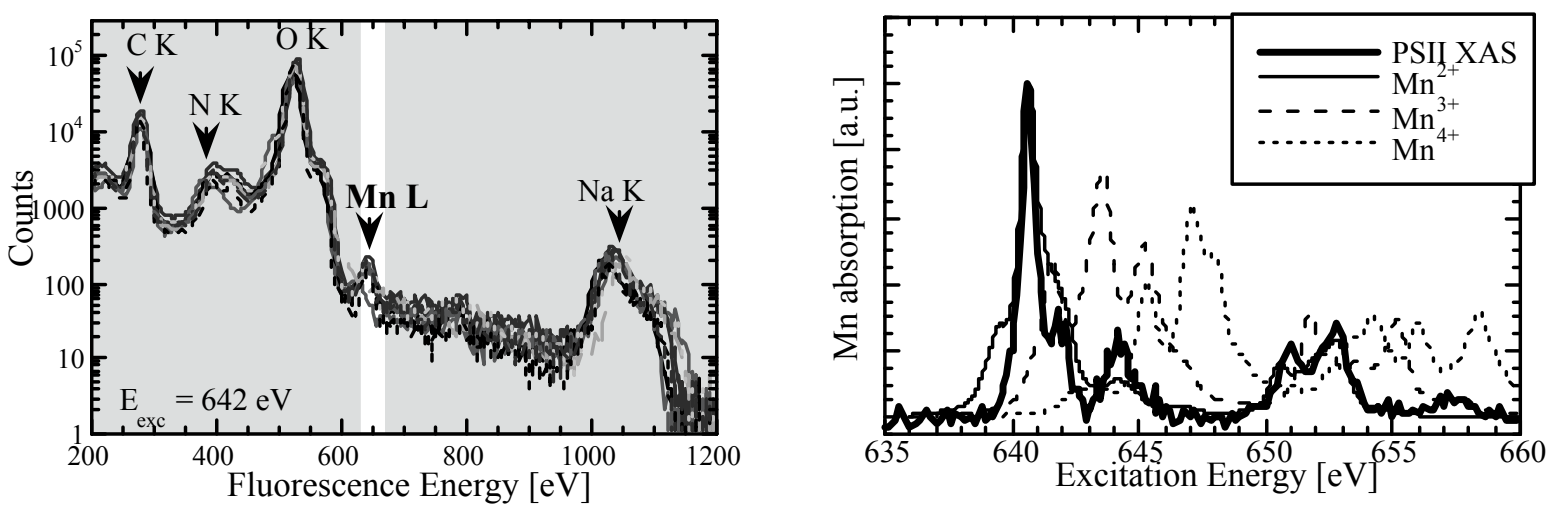

Fig. 1a (top): Fluorescence spectra of PSII taken with a 9-channel cryogenic detector array. 1b (bottom): Mn L-edge absorption spectrum of biologically inactive PSII, and model calculations of the expected edge shifts with Mn oxidation state.

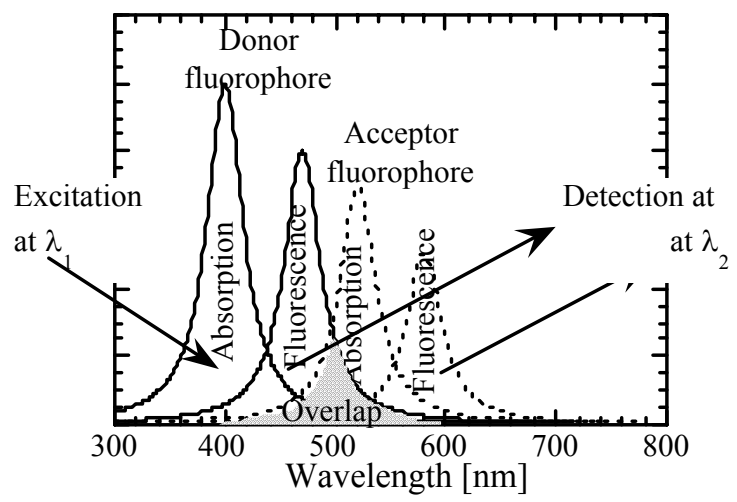

Fig. 2.: Schematic of fluorescence resonance energy transfer. Fluorophores can be designed that change their emission wavelength with environmental conditions. 